\title{
Challenging the uncertainty of COVID-19 in rural community based medical education for family medicine: A thematic analysis
}

Ryuichi Ohta ( $\nabla$ ryuichiohta0120@gmail.com )

Unnan City Hospital

\section{Yoshinori Ryu}

Unnan City Hospital

\section{Chiaki Sano}

Shimane University

\section{Research Article}

Keywords: community-based medical education, COVID-19, family medicine, Japan, rural

Posted Date: December 1st, 2020

DOl: https://doi.org/10.21203/rs.3.rs-109214/v1

License: (c) (i) This work is licensed under a Creative Commons Attribution 4.0 International License.

Read Full License 


\section{Abstract}

\section{Background}

Community based medical education (CBME) enriches undergraduate and postgraduate students' perspectives, especially in rural settings. For the continuity of rural CBME in Japan, the present disruption caused by the coronavirus must be overcome to drive CBME and educate future family physicians adequately in rural community hospitals. In this study, we examine the challenges faced by and solutions for sustainable CBME in community hospitals during the COVID-19 pandemic.

Methods

A qualitative study was performed with CBME stakeholders in a community hospital. First, direct observation was performed by the first author. Through this method, field and reflection notes were taken by observing and interviewing the dean, medical teachers, nurses, medical students, a medical clerk, and rural citizens in the hospital and its surrounding rural communities from April 1 to September 30, 2020. Interviews were recorded, and their contents were transcribed verbatim. The authors analyzed the transcriptions using on a thematic analysis.

Results

Overall, 31 pages of field and reflection notes were collated through direct observation and used for analysis. Five physicians, eight nurses, one clerk, fourteen medical trainees, and three rural citizens were interviewed. Through the thematic analysis, three themes emerged: the uncertainty of COVID-19, an overwhelming fear within medical institutions, and motivation and determination to continue providing CBME.

\section{Conclusion}

Rural CBME for family medicine should be continued for the sustainability of rural medicine, even during the COVID-19 pandemic. The end of the pandemic cannot be foreseen, and CBME in rural areas should be continued to serve its true purpose by carefully considering precautions and through continuous dialogue between stakeholders and medical educators.

\section{Background}

The COVID-19 pandemic has had a tremendous impact on community based medical education (CBME). CBME provides undergraduate and postgraduate students multiple opportunities to learn and broaden their perspectives on medicine, practical knowledge, skills, and attitudes toward doctors, especially in rural settings. ${ }^{1,2,3}$ CBME programs can motivate students to become family physicians and work in rural and remote areas, which is critical for the sustainability of medicine in such areas. ${ }^{4,5}$ In CBME, the collaboration among various stakeholders such as universities, general hospitals, medical teachers, 
medical trainees, other healthcare professionals, and citizens is essential. ${ }^{1,6,7}$ The COVID-19 pandemic, however, has deeply impacted these relationships, primarily because of mutual fear of infection.

Medical institutions fear the transmission of infections from one institution to another and the risk their trainees face in such a situation..$^{8,9}$ Medical trainees may face a lack of essential opportunities for clinical medicine in the institutions they are enrolled in because of reduced involvement by these institutions. 8,10 The continuity of CBME can be vital for the sustainability of rural medicine, as this education is what motivates medical trainees to work at rural medical institutions. ${ }^{11,12}$ For the continuity of CBME, the presently raging fear of medical institutions should be dispelled and interventions made by revising the curriculum of CBME to suit the context of the pandemic.

The COVID-19 pandemic has reduced the efficacy of CBME in Japan. Fear of the pandemic has led to significant reduction in the practical learning experiences that are essential for trainee doctors' development, such as face-to-face interactions with patients and community workers. ${ }^{13}$ The severity of the pandemic varies depending on the density of population and the distance of cities from local points of origin of the pandemic. ${ }^{14,15}$ In rural areas, there are few COVID-19 patients and the standard precautions and protocol against COVID-19 are effective in controlling the infection. ${ }^{14}$ However, presently, rural CBME stands the risk of interference because of social fear of the spread of infections in urban areas, on one hand, and rural citizens' mental stress from self-quarantine, which can complicate the rural education of family medicine trainees, on the other. ${ }^{10,16}$

The presently adverse situation must be overcome to drive CBME and educate future family physicians adequately in rural community hospitals. ${ }^{17}$ To continue delivering rural CBME, the perceptions on limitations and drivers of education should be inquired from stakeholders involved in rural CBME. ${ }^{10}$ Therefore, our research question is, "How do stakeholders of rural CBME consider the continuity of the education in community hospitals?" As this pandemic could persist for months or years, clarifications from the stakeholders about their ideas on and establishment of reasonable solutions for the continuity

of rural CBME are critical for the sustainability of rural medicine. ${ }^{12}$ To date, there has been no study of stakeholders' perceptions of the continuity of rural CBME during the COVID-19 pandemic. This study investigates the challenges posed by the COVID-19 pandemic to and the possible solutions for sustainable CBME in community hospitals.

\section{Methods}

\section{Setting}

Unnan is a small, remote, rural city in Japan. In March 2020, the total population was $37,637(18,145$ males and 19,492 females), of whom $39 \%$ were aged over 65 . The city has 16 clinics, 12 homecare stations, 3 visiting nurse stations, and 1 public hospital. At the time the study was conducted, Unnan City Hospital had 281 care beds. The hospital had 27 physicians, 197 nurses, 7 pharmacists, 15 clinical technicians, 37 therapists, 4 nutritionists, and 34 clerks. Most hospital health workers were from the city. 


\section{Rural CBME in Unnan City Hospital}

In the CBME in Unnan City Hospital, medical trainees work with family doctors at the community hospital and the affiliated clinic to learn about the most frequently reported illnesses and how they were managed through systematic practice and person-centered care, a comprehensive and integrative approach. They collaborated with care managers and home care workers to learn about inter-professional work, a competency expected of family doctors. In community care settings, they participated in activities to learn about rural people, such as discussions of public health from the perspectives of person-centered care and community orientation. To promote their learning, the participants reflected on their performance through 10- to 15-minute-long discussions with their teachers at the end of each day. ${ }^{18}$

\section{Participants}

The participants were rural CBME stakeholders at a Japanese rural community hospital that educates more than 40 trainees in family medicine every year. ${ }^{18}$ Participants included physicians (the dean, the director of the hospital, and medical educators), nurses (the director of nursing, deputy directors, head nurses), clerks, and citizens collaborating in the CBME.

\section{Measurement}

First, direct observation was performed by the first author. The first author was a family physician who has worked and been in-charge of medical education in the rural community hospital for five years. The author observed the clinical situations at and medical education of undergraduate and postgraduate trainees in a rural community hospital in Unnan from April 1 to May 31,2020. Field notes were taken through direct observation and by conversing with the dean, medical doctors, medical teachers, nurses, medical students, a medical clerk, and rural citizens in the hospital and surrounding rural communities. Field notes focused on the interactions among healthcare professionals, citizens, and medical trainees concerning the trainees' behaviors, conversational style, and medical training at the hospital. One-on-one interviews, each about 20 minutes long, were conducted with each participant from April 1 to September 30,2020 . Interviews were recorded and their contents transcribed verbatim. The authors analyzed the transcriptions based on a thematic analysis. The interview guide included four main questions based on the previous review article ${ }^{20}$. What did you think about when accepting medical trainees in this pandemic? What do you think about the inhibitions of accepting medical trainees in this pandemic? How do you think about the drivers of accepting medical trainees in this pandemic? What do you think about rural CBME for your hospital?

\section{Data Analysis}

The thematic analysis was used to identify the challenges posed by the COVID-19 pandemic to and the possible solutions for sustainable rural CBME in community hospitals in Unnan. ${ }^{21}$ The first and second authors carefully read the field and reflection notes and interview transcriptions. The first author then coded the content and developed codebooks based on repeated reading. ${ }^{21}$ The second author also coded 
the materials and discussed the coding and codebooks with the first author. In this process, the authors inducted, merged, deleted, and refined concepts and themes by comparing research materials and

coding. ${ }^{5}$ Discussion of data and coding continued until mutual agreement was reached and no new concepts and themes emerged. For member checking, the analysis was provided to all participants, whose feedback was included in the final revision of the themes and concepts. Eventually, no new themes emerged during the member checking phase, indicating saturation. Finally, the themes and concepts were discussed and agreed upon by all the authors.

\section{Ethical Considerations}

Before providing written consent, participants were informed that the data would only be used for research purposes. They were also informed about the research aims, data disclosure procedures, and steps taken to protect personal information. This study was approved by the Unnan City Hospital Clinical Ethics Committee.

\section{Results}

Overall, 31 pages of field notes were written down through the direct observation. In all, 6 physicians, 8 nurses, 3 clerks, 14 medical trainees and 3 citizens were interviewed. Through the thematic analysis, three themes emerged: the uncertainty of COVID-19, an overwhelming fear within medical institutions, and motivation and determination to continue providing CBME. Figure 1 depicts the conceptual framework.

Figure 1. The vicious cycle of fear of COVID-19 and the challenge to the continuity of CBME

\section{The uncertainty of COVID-19}

Most COVID-19 cases have been reported in urban populations, and rural areas have had fewer cases. After a state of emergency was declared in Japan, undergraduate and postgraduate medical education institutions became hesitant about exposing their trainees to patients, even when patients showed no COVID-19 symptoms. One of the participants stated,

"In this pandemic, there are many patients with no symptoms, and young patients are spreading their infections in urban areas. So, medical trainees can be transmitters of the infection even in rural areas. At first, we did not welcome medical trainees." (Physician 5)

An abundance of caution was observed in rural settings, where, ironically, COVID-19 was not a major concern. Medical professionals in the community hospital feared the ambiguity and risk of the infections. One of the participants stated,

"The present situation is complicated because of a lot of unknown things regarding COVID-19. There are various rumors about the transmission of COVID-19, which can cause anxiety among medical staff and citizens." (Nurse 1) 
CBME for medical trainees was not accommodated, even in rural Japanese settings. Communities served by a rural hospital did not have many COVID-19 patients. COVID-19 can be transmitted via air droplets and asymptomatic patients can spread the virus to others, though no studies have conclusively reported that asymptomatic COVID-19 carriers transmit the virus to others. One of the participants stated,

"This infection has many unknowns for us. So, we tend to feel more fear than what is real. The fear may overburden our usual work with additional activities, such as in medical education." (Clerk 1)

The rapid spread of the infection changed the role of the rural hospital, which had to quickly prepare itself for accepting COVID-19 patients. The rapid change in the hospital's role left the healthcare professionals exhausted. In addition, despite low risk, medical education activities were restricted by the rural Unnan City Hospital administration due to concerns that trainees could be exposed to infected patients outside of the rural city. Rural healthcare professionals hesitated to accept people from different places including medical trainees. One of the participants stated,

"Our hospital's function may change through this pandemic, although we do not know our hospital's role clearly. However, we have to prepare for changes. In such a situation, education of trainees can be excluded from the mainstream hospital administration activities." (Physician 3)

\section{Overwhelming fear in medical institutions}

The pandemic has perplexed medical educators and administrators of institutions that engage in medical education. With public accusations of COVID-19 transmissions among the staff of medical institutions, administrators are apprehensive about involving medical students in patient management. The fear among stakeholders, such as deans of teaching hospitals, healthcare professionals, and the general public, has resulted in the inhibition of CBME. There is a perception that even a single COVID-19 case could cause the conditions and reputations of hospitals and other medical institutions to deteriorate. This has led to unfavorable circumstances, such as the Unnan City Hospital being unwilling to accept medical trainees and residents. Some of the participants stated,

"COVID-19 appeared suddenly, and our rural communities had several cases. We cannot focus on the future of COVID-19 clearly. If there is a transmission of this infection to our staff, we may have to close our hospital. So, we tend to hesitate to accept medical trainees from other institutions." (Clerk 2)

"We are feeling the rejection from the rural communities. We do not have any symptoms, and there are no patients in our prefecture. However, just coming from urban areas can trigger people's fear against COVID19, which can inhibit our acceptance of learning in communities." (Medical trainee 7)

The social fear which has been induced by COVID-19 has been getting stronger while the number of the patients has been correspondingly increasing. In rural areas, however, because there were few COVID-19 patients, rural citizens might not have felt so frightened about COVID-19 and thus have not been taking appropriate precaution. However, as social fear spread and other citizens' perceptions began to be considered, they became hesitant about accepting medical trainees. One of the participants stated, 
"Rural social norm and fear is persistent even when there are no patients with COVID-19. We can accept medical trainees, and they can motivate us to work in communities by being interested in our lives and traditions. However, when thinking about our continuity of lives, accepting medical trainees can be a risk." (Citizen 2)

In rural CBME, medical trainees learn in community activities to motivate rural citizens to live actively. During this pandemic, while there were some activities in rural communities and though rural citizens suggested that medical trainees could participate in these activities, the community hospital hesitated to let medical trainees learn through community activities. Rural hospital staff involved in CBME feared the possibility of medical trainees transmitting the infection to rural citizens, which could deteriorate the reputation of the hospital. One of the participants stated,

“Community rumors can affect our hospital's reputation. In social media, hospitals with intra-hospital infection of COVID-19 were criticized drastically, and our hospital could be next. Many stakeholders in our hospital may hesitate to accept medical trainees and provide CBME." (Clerk 1)

The situation of this pandemic has changed drastically due to continuous new findings, and the uncertainty of COVID-19 causes the fear in the stakeholders of rural CBME. The fear induced various worse prediction for the future education in rural institutions like vicious cycle. This cycle inhibited the provision of CBME in rural medical institutions.

\section{Motivation and determination}

The Unnan City Hospital is dependent on physicians who are educated there-it is important to ensure that medical students continue to be trained as family physicians. Through rational discussions about family medicine and the risk of COVID-19 infection, stakeholders acknowledged that inhibiting CBME may reduce the number of family physicians, which would further propel fear. Some of the participants stated,

"We have to prepare for the situations that may arise after this pandemic, and medical education of medical trainees can be important for the future of the hospital and the rural communities." (Physician)

"There are some risks of infection in this era. However, the risk of losing future medical resources should be considered regarding not only physicians but also nurses." (Nurse 4)

The Unnan City Hospital was determined to proceed with its CBME program because it was deemed necessary for the sustainability of the community's healthcare. One of the participants stated,

"Medical educators and trainees' motivation can be respected for the hospital's future. Our CBME can improve the hospital's medical care and lead to an increase in the number of family physicians. This pandemic may prolong further, and the continuity of CBME should be discussed to ensure sustainable community care." (Nurse 6) 
Medical educators and trainees were determined to maintain their health and learning during this pandemic. This was encouraged through continuous discussion among stakeholders regarding the benefits of CBME for family medicine. Involving stakeholders in CBME provision was vital for the sustainability of CBME at the Unnan City Hospital. ${ }^{22}$ The participants stated,

"In rural CBME, we can experience a lot of clinical situations and learn the importance of family medicine and primary care. In the situation of university and general hospital education, clinical experience in rural hospitals is vital for medical trainees to increase clinical experiences." (Medical trainee 5)

"As medical educators, we can educate medical trainees through collaboration with various stakeholders. This time, we hesitated to accept medical trainees initially, but thinking about the future of rural communities, we saw CBME can be critical for sustaining medical resources." (physician 5)

This also resulted in standards being set for accepting medical trainees and the extent to which the trainees could interact with patients, based on the severity of the pandemic. The driver of this discussion was the realization of the importance of CBME for the hospital's future. Medical educators at the rural City Hospital recognized the limitations of $\mathrm{CBME}$ in this pandemic but remained strongly motivated to educate trainees. Everyone involved developed a strong determination to continue CBME. One of the participants stated,

"For our hospital's future, CBME should be continued. This education is sustaining our hospital's function. For the provision of CBME during this pandemic, we have to share the vision of CBME in this hospital. The provision of CBME for medical trainees and our hospital's future should be established through a discussion among stakeholders." (Physician 1)

\section{Discussion}

This study shows the present conditions of rural CBME and the need for sustaining CBME in rural community hospitals during the COVID-19 pandemic. The lack of evidence for control of COVID-1922 induced various fears among the stakeholders of medical education. It was evident that CBME provision should be determined by both the needs of medical students and universities and the accepting hospital's consideration of its future and that of its communities. ${ }^{23}$ To prepare for future turbulence, all CBME stakeholders should support and reinforce their determination to continue providing CBME.

The fears regarding accepting medical trainees from urban areas do not disappear even if stakeholders' motivation and determination remain strong. COVID-19 has spread all over the world, and researchers are dedicatedly studying this virus, leading to a large volume of scientific publications. New information is relentlessly being disseminated by the mass media with updates on the standards of approaching the infection. ${ }^{24}$ As the participants stated, the situation of the pandemic is changing constantly and the rapidity with which the changes are occurring is overwhelming people. They also deeply feel the risk of infection and becoming carriers of the virus. Based on the participants' statements in this research, accepting medical trainees from urban areas can trigger fear, inhibiting their work by increasing the 
additional work of infection control. The trend of fearing medical trainees can be strong among nonmedical professionals such as medical clerks and care workers because of a lack of medical knowledge. ${ }^{25}$ For the continuity of rural CBME, constant information provisions and mitigation of the stress in non-medical workers should be performed frequently by sharing information about the pandemic and giving clarifications about the amount of the risk of the infection they face from medical trainees based on the statistics.

The balance between rural CBME and the severity of the pandemic is essential for the continuity of rural CBME. There may be several waves of the COVID-19 pandemic, as well as fluctuations in the number of COVID-19 patients and the risk of infection. ${ }^{26}$ As the participants stated, medical educational institutions have to adjust their modes of teaching to the situation of the pandemic. Even as rural social norms should be respected during the pandemic, the dialogue with various stakeholders is essential for the continuity of CBME. ${ }^{23}$ Besides, the analysis of this study shows that rural CBME can be critical for medical trainees to gain experience in primary care and family medicine. The establishment of the standard of accepting medical trainees and their range of clinical trainings allowed in community hospitals should be discussed among the stakeholders involving medical trainees themselves. ${ }^{27}$ This process can motivate medical trainees and medical universities that allocate students in institutes offering CBME to consider concrete measures to provide rural CBME.

Stakeholders' motivation and determination can be sustained by the continuous discussion of the importance of rural CBME for the sustainability of rural medicine. Through this pandemic, various stakeholders had experienced tremendous difficulties in imparting rural CBME. ${ }^{27}$ Based on these results, deans and clerks in community hospitals had to negotiate with hospitals and universities that allocate medical trainees to community hospitals for the criteria of acceptance of medical trainees. Referring to the previous article related to our context of the pandemic of COVID-19, medical trainees and medical teachers struggled to learn clinical reasoning due to the corruption of pretest probabilities of diseases because of the fear of the COVID-19. ${ }^{16}$ As the participants stated, rural nurses also struggled with constructing the system of infection control for COVID-19 because they were not used to performing infection control while accepting medical trainees. Each stakeholder in rural CBME had to focus on different issues in this pandemic. For the effective continuity of rural CBME, their continuous dialogue and discussion regarding rural CBME, respecting each stakeholder's difficulties and ideas, was essential. ${ }^{7,12}$ In fact, their mutual understanding and effective collaboration in the administration of rural CBME can drive the smooth adaptation of the education system to the situation of this pandemic. ${ }^{27}$ Furthermore, during the discussion and dialogue, the importance of rural CBME for the sustainability of rural medicine was discussed, which increased the realization of motivation and determination toward imparting rural CBME.

A limitation of this study is transferability. This research was performed in a rural Japanese community hospital. For application to other settings, this study describes the educational system in depth. Also, in this pandemic, rural hospitals and CBME can be distorted, and our results can be applicable to the 
continuity and management of their CBME systems. Another limitation is confirmability. This research was performed mainly by a medical educator in the community hospital. The relationship between the interviewer and interviewees might have affected the contents of the interviews. To mitigate these limitations, the first researcher discussed the contents with the third author, who is a specialist in infection control from outside the hospital.

\section{Conclusion}

CBME for family medicine should be continued to promote the sustainability of rural medicine, even during the COVID-19 pandemic. The end of the pandemic cannot be foreseen, and CBME in rural areas should continue to be undertaken to serve its true purpose. CBME should be continued while carefully considering precautions and through continuous dialogue between stakeholders and medical educators.

\section{Declarations}

\section{Ethics approval and consent to participate}

Before providing written consent, participants were informed that the data would only be used for research purposes. They were also informed about the research aims, data disclosure procedures, and steps taken to protect personal information. This study adhered to relevant guidelines and regulations. This study was approved by the Unnan City Hospital Clinical Ethics Committee.

\section{Consent for publication}

Not applicable

\section{Availability of data and materials}

The datasets used and/or analyzed during the current study are available from the corresponding author on reasonable request.

\section{Competing interests}

The authors have no competing interests to declare.

\section{Funding}

The authors declare that no financial support was received for this research.

\section{Authors' contributions}

RO designed, collected, and analyzed data, and prepared and revised the manuscript. YR designed, collected, and analyzed data. CS analyzed and revised the manuscript. All authors read and approved the final manuscript. 
Acknowledgements

We would like to thank all the participants who took part in this research.

\section{References}

1. Worley P, Silagy C, Prideaux D, Newble D, Jones A. The parallel rural community curriculum: an integrated clinical curriculum based in rural general practice. Med Educ. 2000;34(7):558-65. Doi: 10.1046/j.1365-2923.2000.00668.x

2. Pagatpatan CP, Jr., Valdezco JAT, Lauron JDC. Teaching the affective domain in community-based medical education: A scoping review. Med Teach. 2020;42(5):507-14. Doi: 10.1080/0142159X.2019.1707175

3. Mennin S, Mennin R. Community-based Medical Education. Clin Teacher 2006;90-6. Doi: 10.1111/j.1743-498X.2006.00093.x

4. Maley $M$, Worley $P$, Dent $J$. Using rural and remote settings in the undergraduate medical curriculum: AMEE Guide No. 47. Med Teach. 2009;31(11):969-83. Doi: 10.3109/01421590903111234

5. Worley P, Prideaux D, Strasser R, Magarey A, March R. Empirical evidence for symbiotic medical education: a comparative analysis of community and tertiary-based programmes. Med Educ. 2006;40(2):109-16. Doi: 10.1111/j.1365-2929.2005.02366.x

6. Worley P. Integrity: the key to quality in community-based medical education? (Part Two). Educ Health (Abingdon). 2002;15(2):129-38. Doi: 10.1080/13576280210133053

7. Worley P. Relationships: a new way to analyse community-based medical education? (Part one). Educ Health (Abingdon). 2002;15(2):117-28. Doi: 10.1080/13576280210133062

8. Lee YM, Park KD, Seo JH. New paradigm of pediatric clinical clerkship during the epidemic of COVID19. J Korean Med Sci. 2020;35(38):e344. Doi: 10.3346/jkms.2020.35.e344

9. Rose S. Medical student education in the time of COVID-19. JAMA. 2020;323:2131-2. Doi: 10.1001/jama.2020.5227

10. Aslan D, Sayek I. We Need to Rethink on Medical Education for Pandemic Preparedness: Lessons Learnt From COVID-19. Balkan Med J. 2020;37(4):178-9. Doi:

10.4274/balkanmedj.galenos.2020.2020.4.002

11. Kelly L, Walters L, Rosenthal D. Community-based medical education: is success a result of meaningful personal learning experiences? Educ Health (Abingdon). 2014;27(1):47-50. Doi: $10.4103 / 1357-6283.134311$

12. Somporn P, Ash J, Walters L. Stakeholder views of rural community-based medical education: a narrative review of the international literature. Med Educ. 2018;52(8):791-802. Doi: $10.1111 /$ medu. 13580

13. Singh R, Subedi M. COVID-19 and stigma: social discrimination towards frontline healthcare providers and COVID-19 recovered patients in Nepal. Asian J Psychiatr. 2020;53:102222. Doi: 
10.1016/j.ajp.2020.102222

14. Ranscombe P. Rural areas at risk during COVID-19 pandemic. Lancet Infect Dis. 2020;20(5):545. Doi: 10.1016/S1473-3099(20)30301-7

15. Verhoeven V, Tsakitzidis G, Philips H, Van Royen P. Impact of the COVID-19 pandemic on the core functions of primary care: will the cure be worse than the disease? A qualitative interview study in Flemish GPs. BMJ Open 2020;10:e039674. Doi: 10.1136/bmjopen-2020-039674

16. Ohta R, Nishikura N, Sano C. Impact of COVID-19 on the analytical diagnosing ability of family medicine residents. J Gen Family Med. 2020. Doi: 10.1002/jgf2.393

17. Ahmed H, Allaf M, Elghazaly H. COVID-19 and medical education. Lancet Infect Dis. 2020;20:777-8. Doi: 10.1016/S1473-3099(20)30226-7

18. Ohta R, Ryu Y, Katsube T, Moriwaki Y, Otani J. Students' perceptions of general medicine following community-based medical education in rural Japan. J Gen Family Med. 2019;20(6):236-43. Doi: $10.1002 /$ jgf 2.274

19. Braun V, Clarke V. Using thematic analysis in psychology. Qual Res Psychol. 2006;3(2):77-101. Doi: 10.1191/1478088706qp063oa

20. Gordon M, Patricio M, Horne L, Muston A, Alston SR, Pammi M, et al. Developments in medical education in response to the COVID-19 pandemic: a rapid BEME systematic review: BEME Guide No. 63. Med Teacher. 2020;42:1202-15. Doi: 10.1080/0142159X.2020.1807484

21. Stollenwerk D, Kennedy LB, Hughes LS, O'Connor M. A systematic approach to understanding and implementing patient-centered care. Fam Med. 2019;51(2):173-8. Doi: 10.22454/FamMed.2019.320829

22. Tashiro A, Shaw R. COVID-19 Pandemic response in Japan: what is behind the initial flattening of the curve? Sustainability. 2020;12(13):5250. Doi: 10.3390/su12135250

23. Howe A. Twelve tips for community-based medical education. Med Teacher. 2009;24:9. Doi: $10.1080 / 00034980120103423$

24. Llewellyn S. Covid-19: how to be careful with trust and expertise on social media. BMJ. 2020;368:m1160. Doi: 10.1136/bmj.m1160

25. Greenberg N, Docherty M, Gnanapragasam S, Wessely S. Managing mental health challenges faced by healthcare workers during covid-19 pandemic. BMJ. 2020;368:m1211. Doi: 10.1136/bmj.m1211

26. Vannabouathong C, Devji T, Ekhtiari S, Chang Y, Phillips SA, Zhu M, et al. Novel Coronavirus COVID19: current evidence and evolving strategies. J Bone Joint Surg. 2020;102(9):734-44. Doi: 10.2106/JBJS.20.00396

27. Yang D-Y, Cheng S-Y, Wang S-Z, Wang J-S, Kuang M, Wang TH, et al. Preparedness of medical education in China: lessons from the COVID-19 outbreak. Med Teacher. 2020;42:787-90. Doi: $10.1080 / 0142159 \times .2020 .1770713$

\section{Figures}




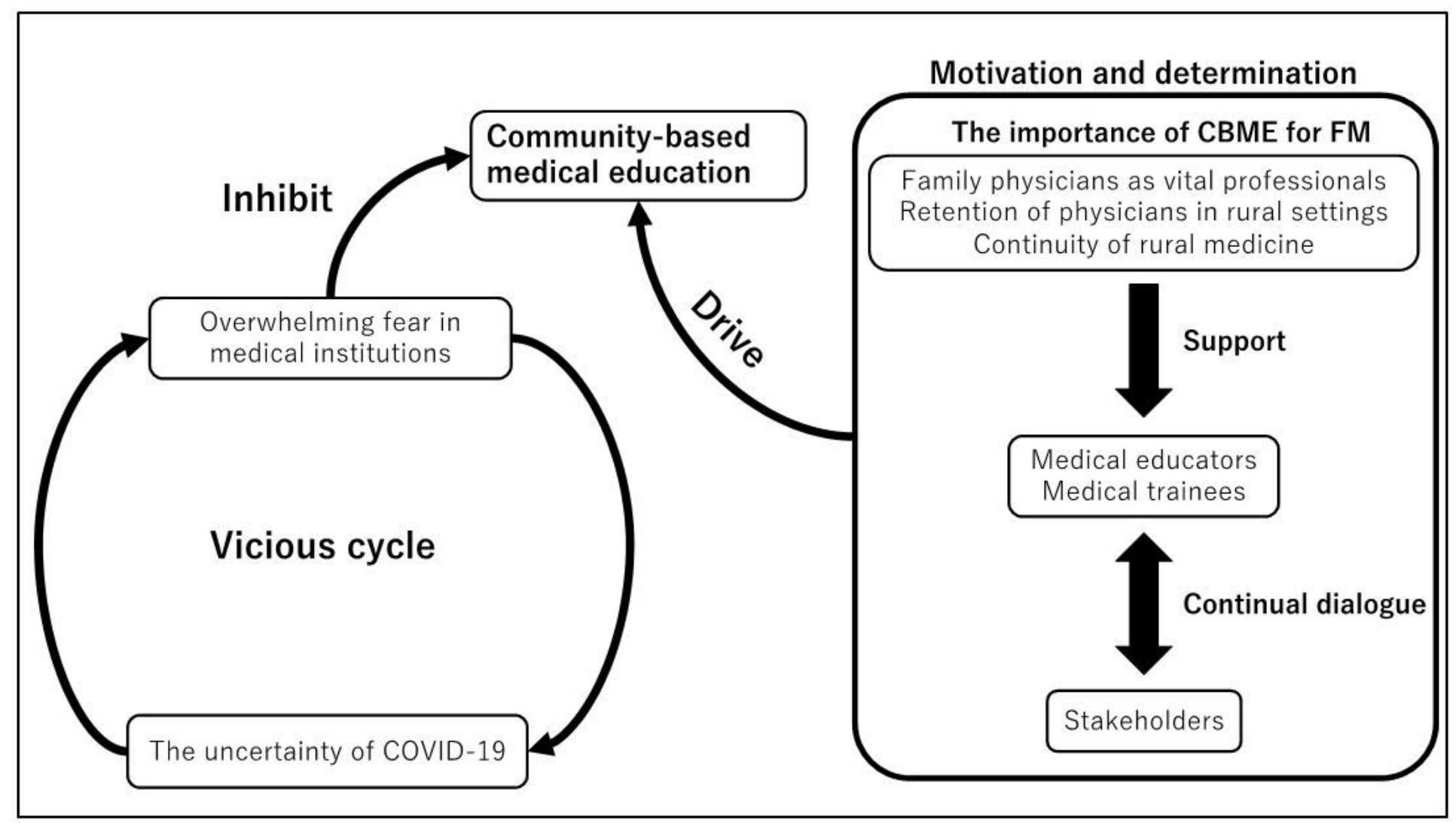

Figure 1

The vicious cycle of fear of COVID-19 and the challenge to the continuity of CBME

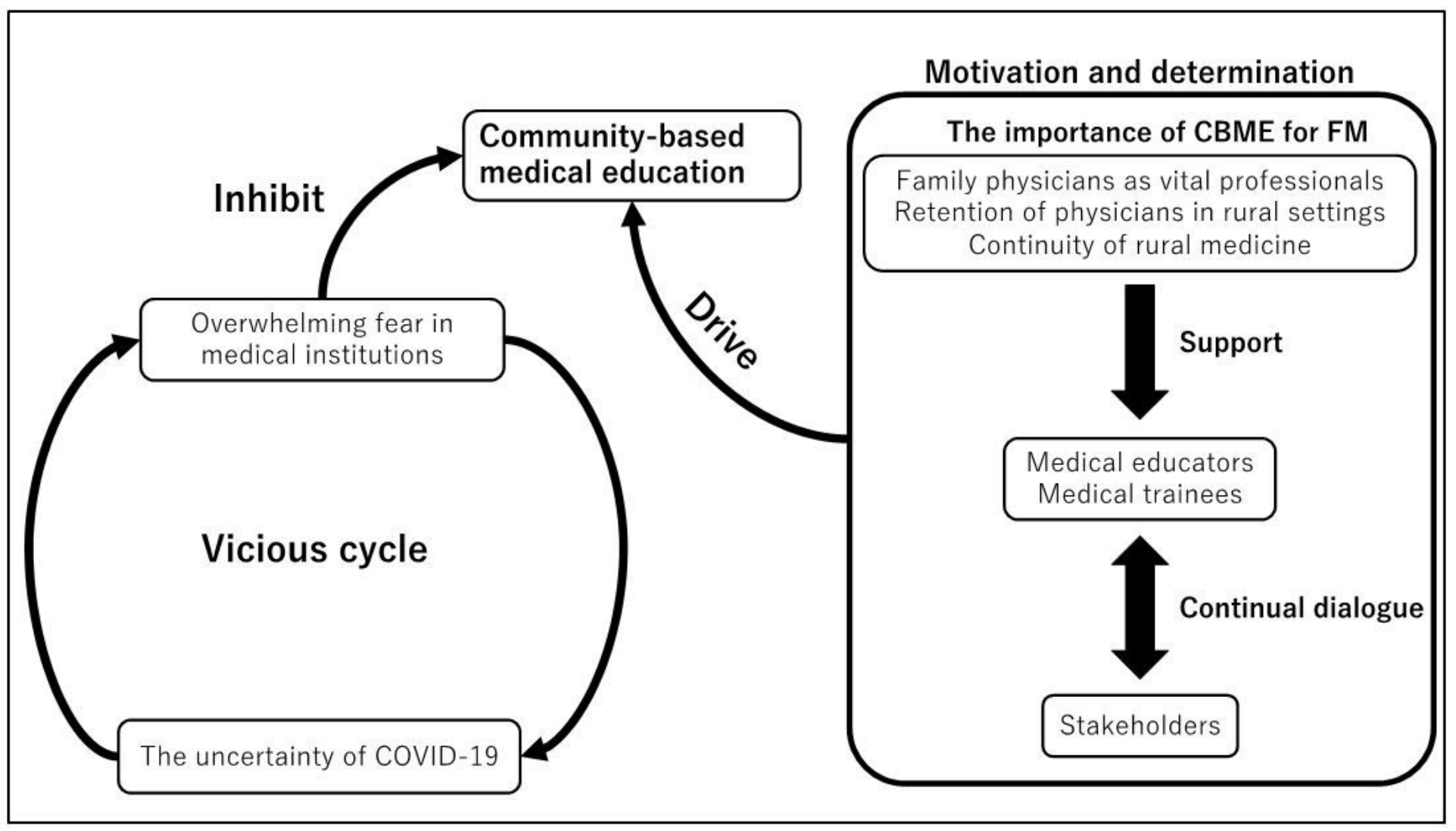

Figure 1 
The vicious cycle of fear of COVID-19 and the challenge to the continuity of CBME 